\title{
Multi-objective Optimal Longitudinal Flight Control System Design for Large Flexible Transport Aircraft
}

\author{
Sipeng Song, James F. Whidborne, Mudassir Lone, Arturo Molina-Cristobal \\ Centre for Aeronautics \\ Cranfield University \\ Bedfordshire, United Kingdom
}

\begin{abstract}
This paper presents a multi-objective evolutionary algorithm design of a longitudinal optimal controller for a large flexible transport aircraft. The algorithm uses a mixed optimization approach based on a combination of Linear Quadratic Regulator (LQR) control and a Multi-Objective Genetic Algorithm (MOGA) to search over a set of possible weighting function structures and parameter values in order to satisfy a number of conflicting design criteria. The proposed approach offers a number of potential optimal solutions lying on or near the Pareto optimal front of competing objectives. The approach is explained in this paper and some results are presented.
\end{abstract}

\section{INTRODUCTION}

Increasing demand for efficiency in the civil aerospace sector has resulted in manufacturers producing highly optimised aircraft with numerous features to reduce the airframe weight. The use of modern materials along with optimised structural designs has helped achieve incremental improvements in performance, but resulted in wings with increasing aspect ratios. This increase in the slenderness of wings has emphasised the importance of aeroservoelasticity where the interactions between the structures, aerodynamics and flight control cannot be neglected. Consequently it is now critical that the flight control system not only provides good handling qualities but also ensures the aeroelastic effects do not have a negative impact on the overall aircraft flight dynamics and passenger comfort.

The problem considered here is the longitudinal handling quality for large flexible aircraft. Handling quality cannot be expressed by a simple criterion or single performance index. A satisfactory design requires consideration of a set of performance indices that are required to meet a set of certification specifications. Thus it is natural to place the problem in a multiobjective setting.

There have been a number of other multi-objective optimization based flight control studies. For example, Tabak et al [1] designed a multi-objective lateral FCS (Flight Control System) based on both fighter aircraft and large manned lifting re-entry vehicle models. The Method of Inequalities (MOI) [2] has been used to design a longitudinal FCS focused on the ride qualities of a Short Take Off and Landing (STOL) aircraft [3]. More specifically, there have been several multiobjective optimization approaches applied to flexible aircraft. These include a multiobjective method based on eigenstructure assignment [4] and $Q$-parameterization closed loop shaping approaches [5], [6].
Many of the methods proposed for multiobjective control design rely either on a direct search of the controller parameters [1], [2], [7] or a $Q$-parametrization of the controller [5], [6], [8] that maintains convexity of the system performance indices. The former suffer from the problem of multiple local minima and a high dimension search space (except for simple SISO controllers), whilst the latter results in high dimension, complex controllers. An alternative approach, dubbed mixed optimization [9] overcomes some of these limitations by using a numerical method to search for the parameters of the weighting functions required by analytical approaches such as LQR and $\mathcal{H}_{\infty}$-optimal control. The earliest mixed optimization appears to be Baras et al [10] who developed a Computer-Aided Control Systems Design (CACSD) package, DELIGHT.LQG, that used tuning algorithms for the weights of an LQG problem to satisfy a number of engineering specifications. Other early efforts for linear quadratic control include [11]-[13]. An $\mathcal{H}_{\infty}$-based approach using the MOI has been proposed [14], [15]; this was extended to utilize the MOGA as the searching algorithm [16]. The MOGA has also been combined with LMIs for mixed optimization [17] and applied for robust controller design for aircraft lateral dynamics [18].

One of the most important characteristics of aeroservoelastic systems such as a large aircraft is that they are high order, because the models include not only rigid body dynamics but also the aero-structural dynamics. The simple structure of LQR controllers and their relatively simple computation make them a good candidate for high order system control (see [19] for example). Furthermore, for an $\ell$-input, $n$-state system model with the $Q$ and $R$ weighting matrices set to be diagonal, the number of tuning parameters is $\ell+n-1$. The controller structure is simpler than most other optimal synthesis methods such as $\mathcal{H}_{\infty}$. For tuning the parameters, the MOGA offers a number of advantages over hill-climbing methods, particularly with regards to the local minima. Hence in this work, MOGA is mixed with $\mathrm{LQR}$ control to solve the design problem of a multi-objective optimal controller for the longitudinal dynamics of a large flexible transport aircraft.

The aircraft in this study is a conceptual aircraft with flexible wings and known as the AX-1 [20]. It has been developed by the Dynamics Simulation and Control Group (DSCG) at Cranfield University. The configuration is shown in Figure 1. The aircraft's open loop longitudinal flying qualities have been 


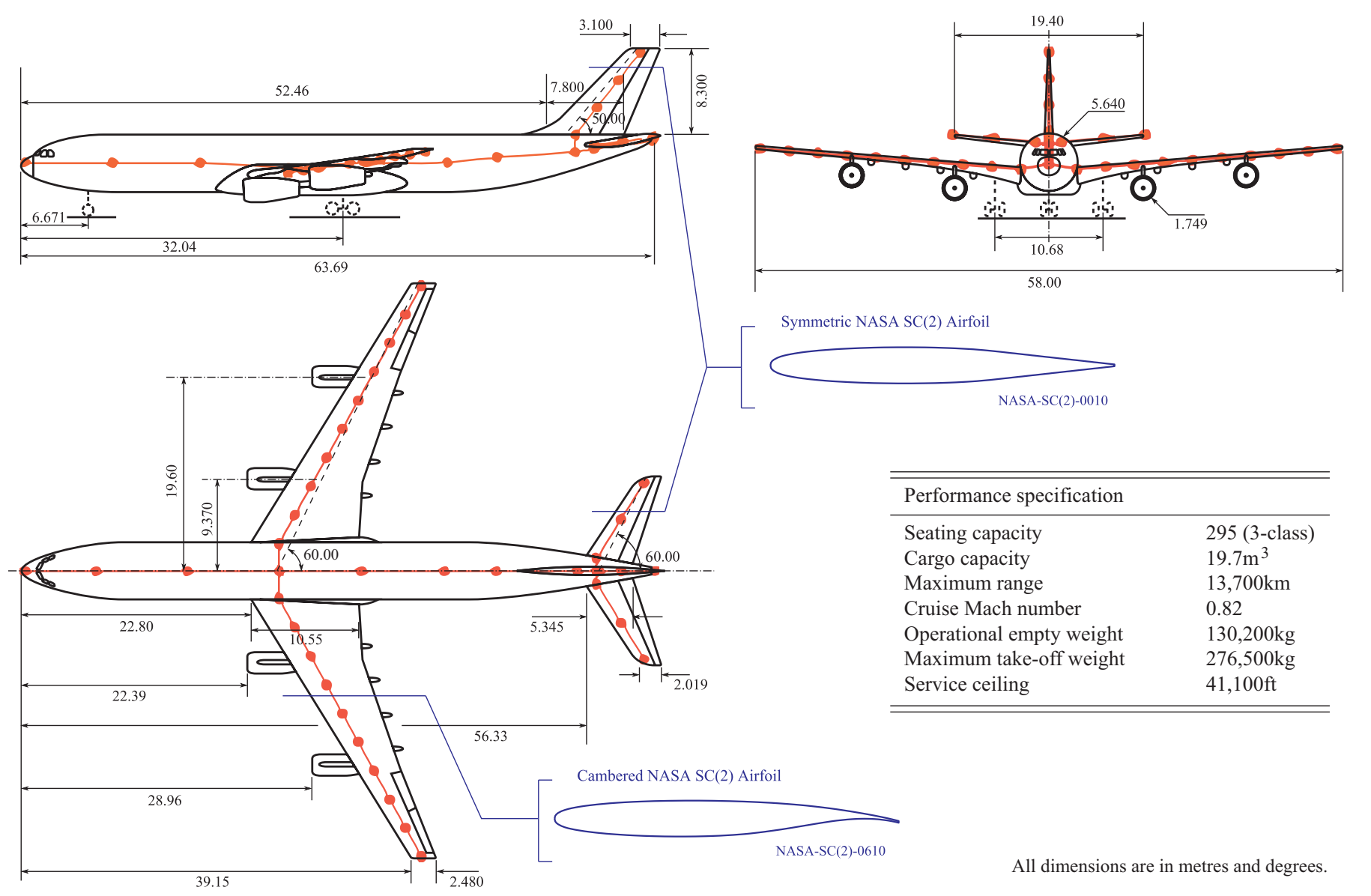

Fig. 1. AX-1 Aircraft Model

shown to be unsatisfactory [21]. For large transport aircraft, the longitudinal performance is also very important for ensuring satisfactory passenger comfort, so this study is confined to the control law design for the longitudinal modes. The system modelling and linearization is summarised in Section II, the theory and algorithm are explained in Section III. Section IV presents the experimental results and, conclusions are in the final section.

\section{SySTEM MODEL}

The AX-1 model is a non-linear generic large transport aircraft model based on the $\mathrm{CA}^{2} \mathrm{LM}$ framework [20], [21]. This model was initially developed for the evaluation of handling qualities of large flexible aircraft. It also provides the capability for flight control law design of user defined airframe configurations.

Most flight control systems consist of a set of flight control laws for different flight conditions and flight missions. In this paper the aircraft is considered at the flight condition with an airspeed of $180 \mathrm{~m} / \mathrm{s}$ and altitude of $20,000 \mathrm{ft}$. The MATLAB linmod routine is used to linearize the model.

The full order linearized state-space model is 33rd order system. There are 9 state variables which are the same as rigid body aircraft, velocities $(u, v, w)$, angular velocities $(p, q, r)$ and Euler angles $(\phi, \theta, \psi)$. The remaining 24 state variables capture 12 different aeroelastic modes with state variables consisting of the structural displacements and corresponding velocities.

In common with most classical fixed wing aircraft, the lateral and longitudinal dynamics can be decoupled and the flight control laws designed separately. There are 4 rigid body longitudinal state variables, $(u, w, q, \theta)$, and the aeroelastic modes can be divided into symmetric and asymmetric. Only the symmetric aeroelastic modes affect the longitudinal responses of the aircraft, so denoting the symmetric aeroelastic mode structural displacements by $\eta_{1}, \ldots, \eta_{6}$, we define the longitudinal dynamics model as

$$
\dot{\mathbf{x}}=\mathbf{A x}+\mathbf{B u}+\mathbf{F w}
$$

with state variable vector, $\mathbf{x}=\left(q, \theta, u, w, \eta_{1}, \ldots, \eta_{6}\right.$, $\left.\dot{\eta}_{1}, \ldots, \dot{\eta}_{6}\right)^{T}$, control $\mathbf{u}=\delta_{e}$ being the elevator deflection and wind disturbance vector, $\mathbf{w}=\left(w_{z}\right)$, which is the wind velocity component in the $z$-direction.

\section{Mixed Optimization Problem Formulation}

\section{A. Mixed Optimization}

If the design aims are expressed quantitatively as a set of $m$ design objective functions $\left\{f_{i}(\mathbf{p}): i=1 \ldots m\right\}$, where $\mathbf{p}$ denotes the design parameter vector chosen by the designer, 
the design problem can be formulated as a multi-objective optimization problem:

$$
\min _{\mathbf{p} \in \mathcal{P}}\left\{f_{i}(\mathbf{p}): i=1 \ldots m\right\}
$$

where $\mathcal{P}$ denotes the set of possible design parameters. In general, the problem (2) is hard to solve if mappings $\mathbf{p} \mapsto f_{i}$ are non convex, particularly if $m$ is not small.

Typically, $\mathbf{p}$ parameterizes the controller as a set of control gains. However, given an $\ell$-input, $n$-state system linearised model,

$$
\begin{aligned}
& \dot{\mathbf{x}}=\mathbf{A x}+\mathbf{B u}, \\
& \mathbf{y}=\mathbf{C x}
\end{aligned}
$$

with control $\mathbf{u}=-\mathbf{K} \mathbf{x}$ and quadratic performance cost function

$$
J=\int_{0}^{\infty}\left(\mathbf{x}^{T} \mathbf{Q} \mathbf{x}+\mathbf{u}^{T} \mathbf{R u}\right) d t
$$

where $\mathbf{Q}$ and $\mathbf{R}$ are are the positive semi-definite control weighting matrices, then the controller that minimizes $J$ is well-known and is given by $\mathbf{K}=\mathbf{R}^{-1} \mathbf{B}^{T} \mathbf{P}$ where $\mathbf{P}=$ $\mathbf{P}^{T}>0$ is found by solving the algebraic Riccati equation $\mathbf{A}^{T} \mathbf{P}+\mathbf{P A}-\mathbf{P B R}^{-1} \mathbf{B}^{T} \mathbf{P}+\mathbf{Q}=0$. If $\mathbf{p}$ parameterizes the weighting matrices $\mathbf{Q}$ and $\mathbf{R}$, then (2) represents a mixed optimization problem which overcomes some of the limitations of the direct approach (see [15]). In this study, the $\mathbf{Q}$ and $\mathbf{R}$ matrices are constrained to be diagonal positive matrices

$$
\mathbf{Q}=\operatorname{diag}\left(q_{1}, \ldots, q_{n}\right) \quad \text { and } \mathbf{R}=\operatorname{diag}\left(1, r_{1}, \ldots, r_{\ell-1}\right) \text {. }
$$

GA's are very-well suited for solving multi-objective optimization problems. In particular the MOGA [22], which is used to solve the problem, is an extension on an idea by [23] which is to develop a population of Pareto-optimal or near Pareto-optimal solutions. This is achieved by finding a set of solutions which are non-dominated. An individual $j$ with a set of objective functions $f^{j}=\left\{f_{1}^{j}, \ldots, f_{m}^{j}\right\}$ is said to be non-dominated if for a population of $N$ individuals, there are no other individuals $k=1, \ldots, N, k \neq j$ such that $f_{i}^{k} \leq f_{i}^{j} \forall i=1, \ldots, m$ and $f_{i}^{k}<f_{i}^{j}$ for at least one $i$. With the MOGA, non-dominated individuals are given the greatest fitness, and individuals that are dominated by many other individuals are given a small fitness. Using this mechanism, the population evolves towards a set of nondominated, near Pareto-optimal individuals. Details of this mechanism are given in [22].

For transport aircraft pilots prefer command of pitch rate rate, so a Rate Command Attitude Hold (RCAH) control flight control system [24] is used with a structure shown in Figure 2. Rather than feedback $\theta$ directly, feedback pitch rate, $q$, is fed back with integral action as part of a PI controller. Thus the LQR optimal controller, $\mathbf{K}$, is partitioned so that $\mathbf{K}=$ $\left[k_{q}, k_{\theta}, \tilde{\mathbf{k}}\right]$ with $k_{q}$ acting on the pitch rate error signal and $k_{\theta}$ on the pitch rate error integral signal as the PI controller. The remaining state variables, $\tilde{\mathbf{x}}=\left(u, w, \eta_{1}, \ldots, \eta_{6}, \dot{\eta}_{1}, \ldots, \dot{\eta}_{6}\right)^{T}$, are fed back through $\tilde{\mathbf{k}}$.

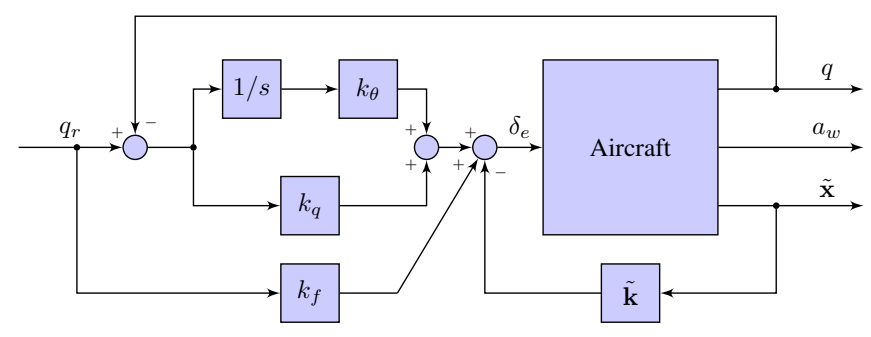

Fig. 2. Controller Structure

A feed-forward gain, $k_{f}$, is included in the controller structure. This gain corrects the reference input, $q_{r}$, so that the output equals the reference input at steady state but only in the short term. Thus the pitch, $\theta$, is removed from the state variables to give a reduced order model with state $\hat{\mathbf{x}}=(q, \tilde{\mathbf{x}})^{T}$ and controller $\hat{\mathbf{k}}=\left[k_{q}, \tilde{\mathbf{k}}\right]$. If $(\hat{\mathbf{A}}, \hat{\mathbf{B}})$ represents the reduced order model matrix pair, then $k_{f}=k_{q}\left(k_{m}-1\right)$ where

$$
k_{m}=\left(\mathbf{c}_{q}(\hat{\mathbf{A}}-\hat{\mathbf{B}} \hat{\mathbf{k}})^{-1} \hat{\mathbf{B}}\right)^{-1}
$$

and $\mathbf{c}_{q}=[1,0, \ldots, 0]$. Output $a_{w}$ in Figure 2 represents the normal acceleration and is used in the next section.

The longitudinal dynamics system is single input, hence $\mathbf{R}$ is a scalar and is set to 10000 . Hence, from (5), the vector of design parameters is

$$
\mathbf{p}=\left(q_{1}, \ldots, q_{n}\right)^{T} .
$$

\section{B. Performance Objective Functions}

A good choice of the design objective functions, $f_{i}$, is critical to allow the correct trade-offs between competing criteria. A main objective is satisfactory longitudinal handling qualities. Here, two objective functions based on the Gibson drop-back criterion [25] are used. Gibson drop-back is based on the response of a low order model that excludes the phugoid mode to a pulse elevator input [26]. Figure 3 shows a typical Gibson drop-back response along with the terms of the Gibson drop-back criteria.

The open-loop test uses a $5^{\circ}$ elevator deflection pulse input as the test input which is typical for flight test. The closed loop reference is pitch rate command, $q_{r}$, and the response is obtained from the system model with the structure of Figure 2 but with a reduced order model that has the phugoid mode removed. This is done by removing the lowest frequency complex mode pair from the Jordan canonical form of the closed-loop system.

Satisfactory handling qualities based on Gibson drop-back can be assessed by two terms, $q_{\max } / q_{\mathrm{ss}}$ and $D B / q_{\mathrm{ss}}$, where the $q_{\mathrm{max}}$ is the maximum value of pitch rate, $q_{\mathrm{ss}}$ is the steady state value of pitch rate for the certain elevator input and $D B$ is the drop-back of the pitch altitude $\theta$, between the maximum value and the steady state value of $\theta$ [25]. The model is linear, hence the input magnitude is unity and the Gibson drop-back input is

$$
q_{r}(t)= \begin{cases}1 & \text { for } t \in\left[t_{0}, t_{1}\right) \\ 0 & \text { for } t \text { elsewhere }\end{cases}
$$




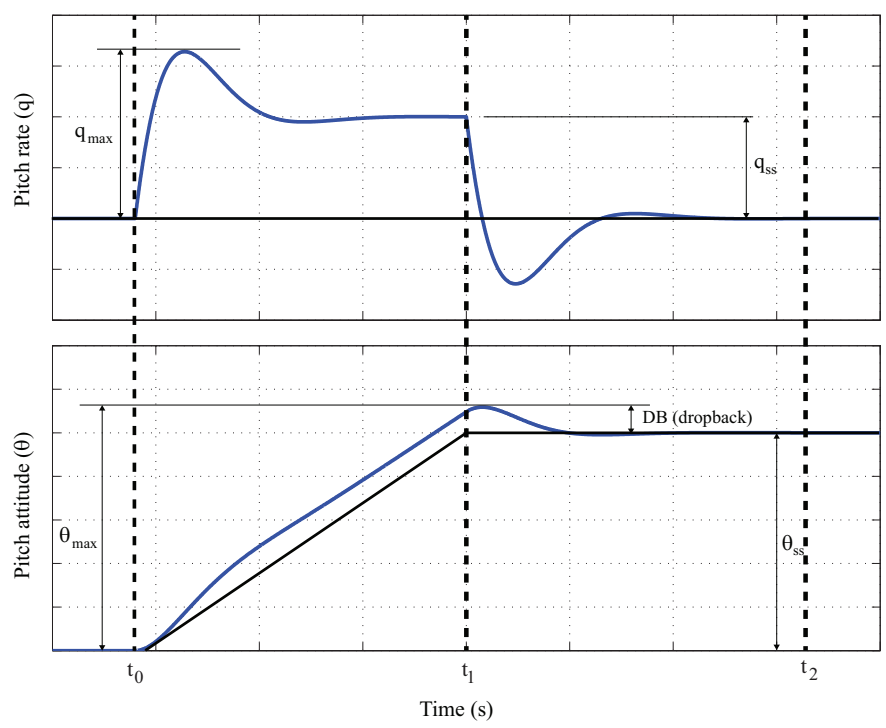

Fig. 3. Definition of $q_{\max }, q_{s s}$ and $D B$ terms

To evaluate $q_{\max }, q_{\mathrm{ss}}$ and $D B$, we take $t_{0}=0$ and we need to calculate $t_{1}$ and $t_{2}$. This is done based on the convergence of the modal responses.

Let $\lambda_{1}, \ldots, \lambda_{n}$ be the eigenvalues of the closed loop system, then if $\alpha$ is the spectral abscissa defined by $\alpha=$ $\max \left\{\operatorname{Re}\left(\lambda_{i}\right): i=1, \ldots, n\right\}$ and let $t_{\mathrm{ss}}=-10 / \alpha$ be the time such that the modal responses have decayed to nearly zero. Then $t_{1}=t_{\mathrm{ss}}$ and $t_{2}=2 t_{\mathrm{ss}}$.

The performance indices used are below.

1) Maximum Pitch Rate: This is the first Gibson criterion,

$$
f_{1}=q_{\max } / q_{\mathrm{ss}},
$$

where $q_{\max }=\max \left\{q(t): t \in\left[t_{0}, t_{1}\right]\right\}$ and $q_{\mathrm{ss}}=q\left(t_{\mathrm{ss}}\right)$.

2) Drop Back: This is

$$
f_{2}=D B / q_{\mathrm{ss}}
$$

where $D B=\theta_{\max }-\theta_{\mathrm{ss}}$ with $\theta_{\max }=\max \left\{\theta(t): t \in\left[t_{0}, t_{2}\right]\right\}$ and $\theta_{\mathrm{ss}}=\theta\left(t_{2}\right)$.

3) Control effort: This is measured by

$$
f_{3}=\left\|T_{u w}\right\|_{2}
$$

where $T_{u w}$ is the closed loop transfer function from $\mathbf{w}$ to $\mathbf{u}$ obtained using (1).

4) Comfort: Passenger comfort during turbulence is also important for a transport aircraft. Kubica and Madelaine [27] developed the following comfort criteria based on the ISO 2631-1 standard

$$
P I P=\frac{1}{3}\left[\int_{0}^{T}\left(a_{w}{ }^{2}(t)\right) d t\right]^{1 / 2}
$$

where PIP means Percentage of Ill Passengers and $a_{w}$ is the normal acceleration. The final time, $T$, is not clearly defined, so here we define the comfort measure as

$$
f_{4}=\left\|T_{a_{w} w}\right\|_{2}
$$

where $T_{a_{w} w}$ is the closed loop transfer function from $\mathbf{w}$ to $a_{w}$
5) Rise Time: This objective function measures the response speed of the system, and ensures the bandwidth is maintained and the system is not over-damped. The rise time is the time taken for the response to rise from $10 \%$ to $90 \%$ of the steady-state response. Figure 4 shows the definition of rise time which is defined as

$$
f_{5}=t_{r}=t_{0.9 q_{\mathrm{ss}}}-t_{0.1 q_{\mathrm{ss}}}
$$

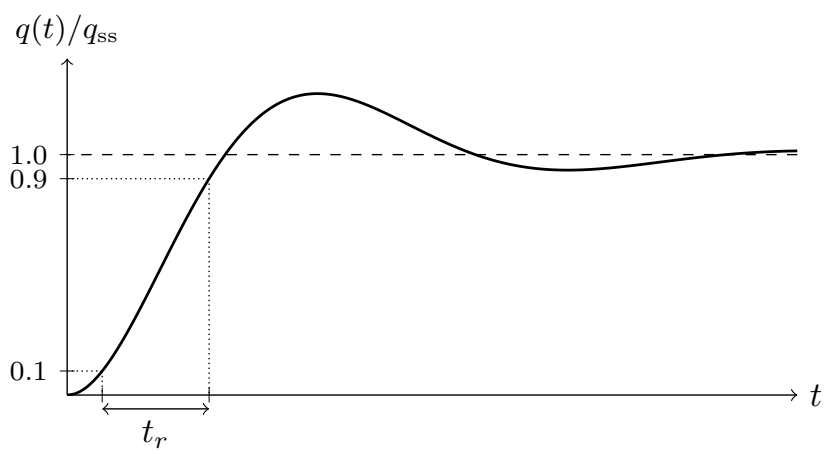

Fig. 4. Definition of Rise Time

\section{Non-linear Constraints}

In addition to the objective functions, the system needs to meet some constraints, in particular a constraint on the maximum controller gain to prevent too great a change from the open-loop dynamics which is neither desirable nor achievable due to actuator saturation. In addition, the flight control law should not overly alter the phugoid mode as this may cause problems with the outer loop autopilot control loops and the calculation of the low-order model required for the calculation of $f_{1}$ and $f_{2}$.

1) Gain Constraint: It is important to limit the gains to reasonable values so the controller can be implemented in practice. Here, the gains are limited in $[-1,1]$ giving the constraint

$$
\|\mathbf{K}\|_{\infty}<1
$$

2) Phugoid Mode Constraint: The phugoid mode is the lowest frequency complex mode pair obtained from the Jordan canonical form of the closed-loop system. For handling quality evaluation, the phugoid mode response is not important. This is because it is comparatively slow compared to the short period mode and does not affect the handling quality. Here, the phugoid mode damping ratio, $\zeta_{\text {phugoid }}$, is constrained so $\zeta_{\text {(phugoid) }} \leq 0.5$. In addition, the phugoid closed-loop poles should not move too far from the open-loop poles, so the mode natural frequency change, $\delta \omega_{n \text { (phugoid) }}$, is constrained so $\left|\delta \omega_{n \text { (phugoid) }}\right| \leq 0.01$.

\section{EXPERIMENTAL RESULTS}

The gamultiobj MOGA from the MATLAB Global Optimization Toolbox was used to search for Pareto optimal solutions of the LQR control weighting represented by $\mathbf{p}$. The algorithm took approximately 45 minutes to evaluate 


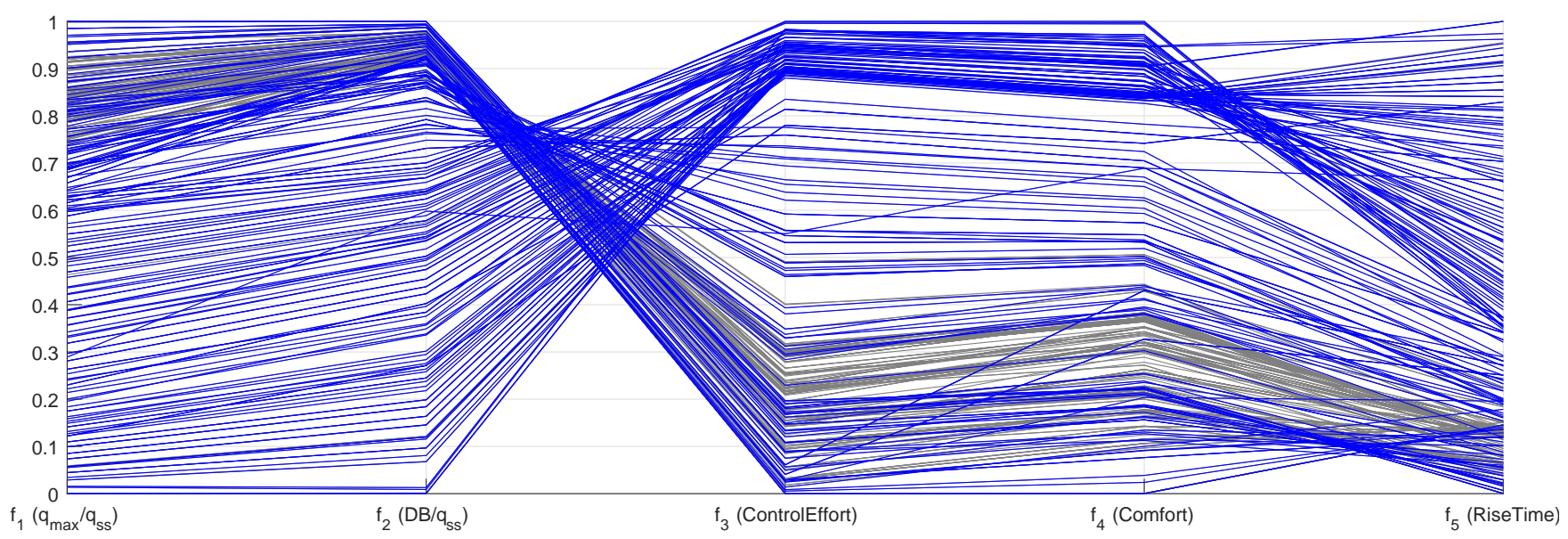

Fig. 5. Multi-objective Trade-off Diagram

102 generations. The final generation's population size is 500 and has 175 non-dominated members. Figure 5 is a tradeoff diagram that shows the relations between the different objectives. The set of non-dominated solutions is represented by the blue lines and the dominated set of solutions by the grey.

The near-Pareto front between $f_{1}$ and $f_{5}$ is shown in Figure 6 . The blue points represent the non-dominated solutions of the final generation and the grey points the dominated solutions. This figure it is not a full-scope Pareto front because of the constraints set previously. The solutions with a rise time less than 0.2 all have low values of the gains on the aeroelastic mode structural displacements $\eta_{1}, \ldots, \eta_{6}$, whereas those with high rise time have high gains on $\eta_{i}$. Two example solutions, a low-gain $\mathbf{p}_{1}$ and hign-gain $\mathbf{p}_{2}$ are chosen. Figure 7 shows the Gibson dropback criteria pitch rate responses for solutions $\mathbf{p}_{1}$ and $\mathbf{p}_{2}$ and the open loop response. The high-gain solution, $\mathbf{p}_{2}$, has a much improved handling qualities response.

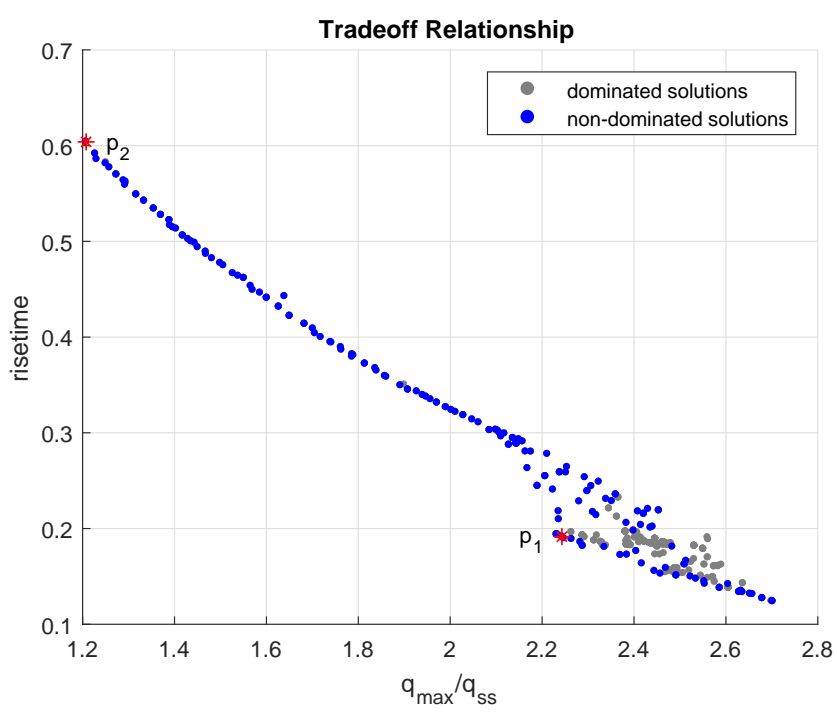

Fig. 6. Pareto Front

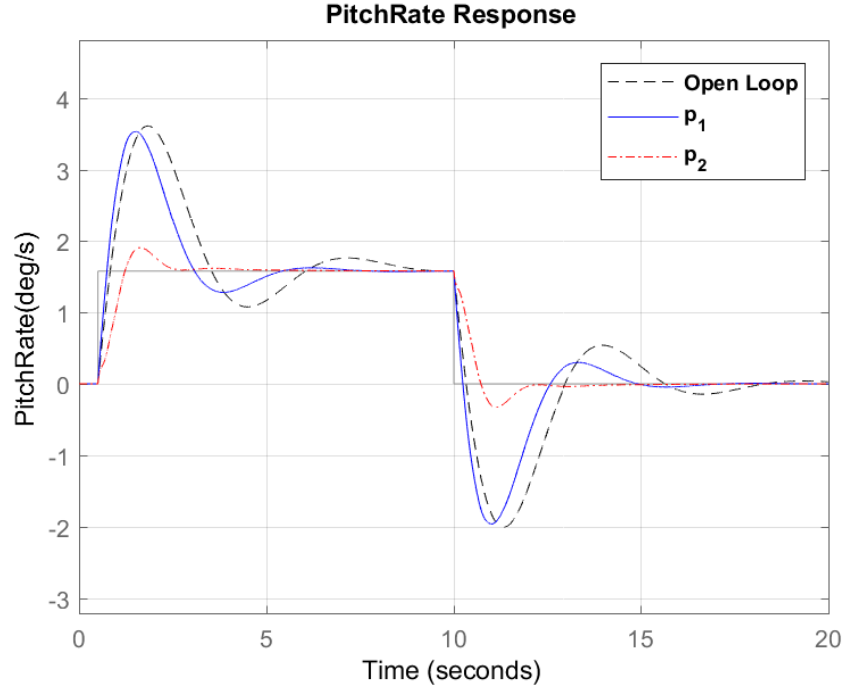

Fig. 7. Gibson Criteria Pitch Rate Response

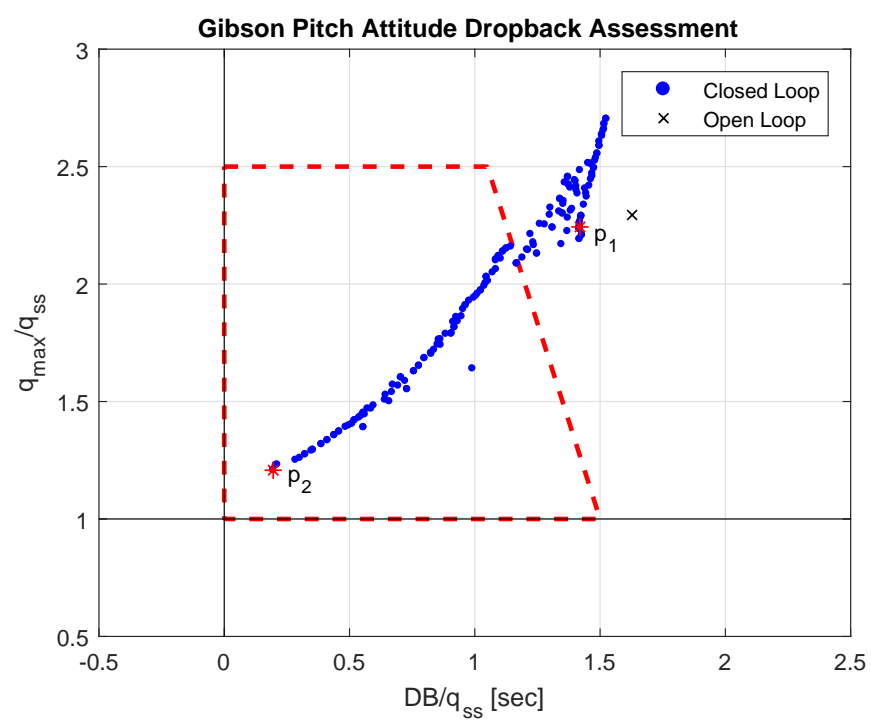

Fig. 8. Gibson Pitch Attitude Drop-back Assessment 
Figure 8 shows the Gibson drop back criteria in a Gibson drop-back assessment diagram [28] for the closed loop systems with the non-dominated solutions shown in Figure 6 and the open loop system. The solution $\mathbf{p}_{1}$ provides better handling qualities than the open-loop system $(\times)$, but is still out of the satisfied region. The solution $\mathbf{p}_{2}$ gives much better performance in handling qualities, but it sacrifices the performance in the other objectives. Note that the gains on the aeroelastic mode structural displacements means the closed-loop system utilizes the information about the structure deformation to achieve a better handling quality performance.

\section{CONCLUSIONS}

The process of a RCAH flight controller design for a large flexible transport aircraft using a multiobjective mixed optimization approach is shown. A variety of objective criteria are used along with an LQG optimal controller. The approach allows trade-off studies to be conducted in order to assist the designer making the right choice of design parameters. However, because of the simple controller structure, the LQR approach does not give a rich set of possible solutions. Further details of the results are available [29].

Several observations can be made. The approach is flexible in its choice of criteria, these included both handling qualities in the form of Gibson drop back criteria as well as a passenger comfort index that measures rejection of gust disturbances and turbulence. The use of an LQR controller with a flexible aircraft is also demonstrated. Further work is needed to validate the controller designs using flight simulation; this can be done through the $\mathrm{CA}^{2} \mathrm{LM}$ framework [20], [21].

The integration of parametric search methods into computer aided control system design tools is now very mature via MATLAB Control System Toolbox tools such as systune and looptune. It would be useful for such tools to also include weighting function parameter tuning in a mixed optimization approach.

\section{REFERENCES}

[1] D. Tabak, A. A. Schy, D. P. Giesy, and K. G. Johnson, "Application of multiobjective optimization in aircraft control systems design," Automatica, vol. 15 , pp. 595-600, 1979.

[2] V. Zakian and U. Al-Naib, "Design of dynamical and control systems by the method of inequalities," Proc. IEE, vol. 120, no. 11, pp. 1421-1427, 1973.

[3] T. R. Crossley and A. M. S. Dahshan, "Design of a longitudinal ridecontrol systems by Zakian's method of inequalities," J. Aircraft, vol. 19, no. 9, pp. 730-738, 1982.

[4] T. Livet, F. Kubica, and J. F. Magni, "Robust flight control design with respect to delays, control efficiencies and flexible modes," Control Eng. Pract., vol. 3, no. 10, pp. 1373-1384, 1995.

[5] G. Puyou, G. Ferreres, C. Chiappa, and P. Menard, "Multiobjective method for flight control law design," in Proc. AIAA Guidance, Navigation, and Control Conference, 2004.

[6] G. Ferreres and G. Puyou, "Flight control law design for a flexible aircraft: Limits of performance," J. Guid. Control Dyn., vol. 29, no. 4, pp. 870-870, 2006.

[7] G. Kreisselmeier and R. Steinhauser, "Application of vector performance optimization to a robust control loop design for a fighter aircraft," Int. J. Control, vol. 37, no. 2, pp. 251-284, 1983.

[8] S. Boyd, C. Barratt, and S. Norman, "Linear controller design: Limits of performance via convex optimization," Proc. IEEE, vol. 78, no. 3, pp. 529-574, 1990.
[9] J. F. Whidborne, I. Postlethwaite, and D.-W. Gu, "A mixed optimization approach to multi-objective computer-aided control system design," in Proc. IEEE Symp. on Comp. Aided Contr. Syst. Design (CACSD'96), Dearborn, MI, 1996, pp. 309-314.

[10] J. S. Baras, M. K. H. Fan, W. T. Nye, and A. L. Tits, "DELIGHT.LQG: A CAD system for control systems design using LQG controller structure,' in 18th Annual Conf. on Inf. Sci and Syst., Princeton, NJ, 1984.

[11] J. E. Paddison, R. M. Goodall, J. Bals, and G. Grübel, "Multi-objective design study for a maglev suspension controller using the databased ANDECS-MATLAB environment," in Proc. IEEE Symp. on Comp. Aided Contr. Syst. Design (CACSD'94), Tuscon, AZ, Mar. 1994, pp. 239-246.

[12] D. Haessig, "Selection of LQR/LTR weighting matrices through constrained optimisation," in Proc. 1995 Amer. Contr. Conf., Seattle, WA, 1995, pp. 458-460.

[13] J. F. Whidborne, G. Murad, D.-W. Gu, and I. Postlethwaite, "Robust control of an unknown plant - the IFAC 93 Benchmark," Int. J. Control, vol. 61, no. 3, pp. 589-640, 1995.

[14] I. Postlethwaite, J. F. Whidborne, G. Murad, and D.-W. Gu, "Robust control of the benchmark problem using $H_{\infty}$ methods and numerical optimization techniques," Automatica, vol. 30, no. 4, pp. 615-619, 1994.

[15] J. F. Whidborne, I. Postlethwaite, and D.-W. Gu, "Robust controller design using $H_{\infty}$ loop-shaping and the method of inequalities," IEEE Trans. Contr. Syst. Technol., vol. 2, no. 4, pp. 455-461, 1994.

[16] N. V. Dakev, J. F. Whidborne, A. J. Chipperfield, and P. J. Fleming, " $H_{\infty}$ design of an EMS control system for a maglev vehicle using evolutionary algorithms," Proc. IMechE, Part I: J. Syst. \& Contr., vol. 311, no. 4, pp. 345-355, 1997.

[17] A. Molina-Cristóbal, I. A. Griffin, P. J. Fleming, and D. H. Owens, "Linear matrix inequalities and evolutionary optimization in multiobjective control," Int. J. Syst. Sci., vol. 37, no. 8, pp. 513-522, 2006.

[18] M. Giacomán-Zarzar, R. Ramirez-Mendoza, P. J. Fleming, I. Griffin, and A. Molina-Cristóbal, "Robust $H_{\infty}$ controller design for aircraft lateral dynamics using multi-objective optimization and genetic algorithms," IFAC Proceedings Volumes, vol. 41, no. 2, pp. 8834-8839, 2008, 17th IFAC World Congress.

[19] J. McKernan, J. F. Whidborne, and G. Papadakis, "Linear quadratic control of plane Poiseuille flow - the transient behaviour," Int. J. Control, vol. 80, no. 12, pp. 1912-1930, 2007.

[20] V. Portapas, A. Cooke, and M. Lone, "Modelling framework for flight dynamics of flexible aircraft," Aviation, vol. 20, no. 4, pp. 173-182, 2016.

[21] V. Portapas, S. Yusuf, M. Lone, and E. Coetzee, "Modelling framework for handling qualities analysis of flexible aircraft," in AIAA Modeling and Simulation Technologies Conference, Grapevive, TX, Jan. 2017.

[22] C. M. Fonseca and P. J. Fleming, "Multiobjective optimization and multiple constraint handling with evolutionary algorithms - part I: a unified formulation," IEEE Trans. Syst. Man \& Cybernetics - A, vol. 28 , no. 1 , pp. 26-37, 1995.

[23] D. E. Goldberg, Genetic Algorithms in Search, Optimization and Machine Learning. Reading, MA.: Addison-Wesley, 1989.

[24] A. McCuish and B. Caldwell, "Development and flight experience of the control laws and the aeroservoelastic solution in the Experimental Aircraft Programme (EAP)," in Advances in Aircraft Flight Control, M. B. Tischler, Ed. London, U.K.: Taylor \& Francis, 1996.

[25] J. C. Gibson, "Criteria for handling qualities of military aircraft,", AGARD, Neuilly-sur-Seine, France, Technical Report AGARD-CP-333, 1982.

[26] E. J. Field, K. F. Rossitto, and D. G. Mitchell, "Landing approach flying qualities criteria for active control transport aircraft," Defense Technical Information Center, Compilation Part Notice ADP011128 RTO MP-051, 2000, presented at the RTO AVT Symposium on "Active Control Technology for Enhanced Performance Operational Capabilities of Military Aircraft, Land Vehicles and Sea Vehicles", Braunschweig, Germany, 8-11 May 2000.

[27] F. Kubica and B. Madelaine, "Passenger comfort improvement by integrated control law design.” Defense Technical Information Center, Compilation Part Notice ADPO10491 RTO-MP-036, 1999.

[28] H. A. Mooij, Criteria for low-speed longitudinal handling qualities. Dordrecht, Netherlands: Springer, 1985, no. NLR TR 83037 U.

[29] S. Song, "Multi-objective optimal longitudinal flight control system design for a large flexible transport aircraft," Master's thesis, Cranfield University, Cranfield, U.K., 2018. 


\section{Multi-objective optimal longitudinal flight control system design for large flexible transport aircraft}

Song, Sipeng

IEEE

Song S, Whidborne JF, Lone M and Molina-Cristobal A. Multi-objective optimal longitudinal flight control system design for large flexible transport aircraft. UKACC 12th International Conference on Control (CONTROL 2018), Sheffield, 5-7 September 2018 https://doi.org/10.1109/CONTROL.2018.8516783

Downloaded from Cranfield Library Services E-Repository 